\title{
Circulating tumor DNA as a marker of treatment response in BRAF V600E mutated non-melanoma solid tumors
}

\author{
Lise Barlebo Ahlborn ${ }^{1,2}$, Ida Viller Tuxen ${ }^{1}$, Florent Mouliere ${ }^{3}$, Savvas Kinalis ${ }^{2}$, Ane \\ Y. Schmidt ${ }^{2}$, Kristoffer Staal Rohrberg ${ }^{1}$, Eric Santoni-Rugiư ${ }^{4}$, Finn Cilius Nielsen ${ }^{2}$, \\ Ulrik Lassen ${ }^{1}$, Christina Westmose Yde ${ }^{2}$, Olga Oestrup ${ }^{2}$ and Morten Mau-Sorensen ${ }^{1}$ \\ ${ }^{1}$ The Phase I Unit, Department of Oncology, Rigshospitalet, Copenhagen University, Copenhagen, Denmark \\ ${ }^{2}$ Center for Genomic Medicine, Rigshospitalet, Copenhagen University, Copenhagen, Denmark \\ ${ }^{3}$ Cancer Research UK Cambridge Institute, University of Cambridge, Cambridge, United Kingdom \\ ${ }^{4}$ Department of Pathology, Rigshospitalet, Copenhagen University, Copenhagen, Denmark \\ Correspondence to: Lise Barlebo Ahlborn, email: lise.barlebo.ahlborn@regionh.dk \\ Keywords: BRAF inhibitor; circulating tumor DNA; mutant allele fraction; early phase study; solid cancer \\ Received: June 04, $2018 \quad$ Accepted: July 29, $2018 \quad$ Published: August 24, 2018 \\ Copyright: Ahlborn et al. This is an open-access article distributed under the terms of the Creative Commons Attribution License \\ 3.0 (CC BY 3.0), which permits unrestricted use, distribution, and reproduction in any medium, provided the original author and \\ source are credited.
}

\section{ABSTRACT}

Purpose: We evaluated longitudinal tracking of BRAF V600E in circulating cellfree DNA (cfDNA) as a marker of treatment response to BRAF inhibitor (BRAFi) combination therapies in non-melanoma solid tumors included in the Copenhagen Prospective Personalized Oncology (CoPPO) program.

Experimental design: Patients with BRAF V600E-mutated tumors were treated with combination therapies including BRAFi. Quantification of mutant cfDNA from plasma was determined and correlated to clinical outcomes. Exome sequencing was performed to identify possible resistance mutations.

Results: Twenty-three patients had BRAF-mutated tumors out of $\mathbf{4 5 5}$ patients included in CoPPO and 17 started BRAFi combination (EGFRi/MEKi) therapy. Tumor responses were achieved in 8 out of 16 evaluable patients and the median overalland progression-free survival (OS and PFS) was 15 and 4.8 months, respectively. Longitudinal measurements of BRAF V600E-mutant cfDNA indicated disease progression prior to radiological evaluation and a reduction in the mutant fraction of more than $50 \%$ after 4 and 12 weeks of therapy was associated with a significantly longer PFS $(p=0.003$ and $p=0.029)$ and $O S(p=0.029$ and $p=0.017)$. Furthermore, the baseline mutant fraction and total level of cfDNA positively correlated with tumor burden $(p=0.026$ and $p=0.024)$. Finally, analysis of cfDNA at progression revealed novel mutations potentially affecting the MAPK pathway.

Conclusion: BRAFi combination therapies showed a response rate of $50 \%$ in BRAF V600E-mutated non-melanoma tumors. The fraction of BRAF-mutant cfDNA represent a sensitive indicator for clinical outcomes with plasma collected at week 4 and 12 as crucial time points for monitoring response and disease progression.

\section{INTRODUCTION}

Activating mutations in the $B R A F$ gene are present in $5-10 \%$ of all human malignancies with the valineto-glutamic acid substitution at codon 600 (V600E) being the far most common mutation [1]. This mutation appears in a wide range of cancers including melanoma [2], colorectal cancer [3, 4], papillary thyroid cancer [5], non-small cell lung cancer $[6,7]$, hairy cell leukemia [8], and cholangiocarcinoma [9]. The introduction of specific 
inhibitors of activated BRAF has greatly improved progression-free survival (PFS) and overall survival (OS) of patients with metastatic melanoma [10]. Dual inhibitor therapies have been successfully tested in malignant melanoma, where combining BRAF inhibitor (BRAFi) treatment with MEK inhibition (MEKi), improved PFS relative to those treated with BRAFi monotherapy [11] and similarly in colorectal cancer adding EGFR inhibitors (EGFRi) to BRAFi [12, 13]. The effect is however temporary as most tumors become resistant to therapy within 6 to 12 months $[14,15]$. Consequently, close monitoring of therapy resistance is required but repeated access to tumor tissue is hampered by the invasive nature of tissue biopsies and the associated complications for the patients.

Circulating cell-free DNA (cfDNA) has the potential to monitor therapy response through a simple blood sample, often referred to as a liquid biopsy. Tumor specific mutations can be identified in cfDNA and hence presents a minimally-invasive strategy to assess tumor material. In solid tumors, cfDNA has shown great promise in cancer diagnosis, monitoring of therapy, and detection of therapy resistance and clonal evolution (reviewed in [16]). Furthermore, early cancer detection and tumor localization were recently demonstrated based on analyses including cfDNA [17]. In advanced malignant melanoma the $B R A F$ $\mathrm{V} 600 \mathrm{E}$ mutation can be detected in up to $84 \%$ of patients with mutation-positive tumors and the level of mutant DNA (cfBRAFV600E) has been shown to reflect response to targeted treatment including progression and tumor burden [18-20]. Most studies have focused on malignant melanoma and to some extent colorectal cancers [18, 2125 ], but little is known about the importance of circulating BRAF DNA in other cancers and how this information is related to BRAFi combination therapy.

In this study, we longitudinally measured the fraction of cfBRAFV600E in plasma samples collected every four weeks from baseline until disease progression from a heterogeneous group of advanced solid cancers included in the Copenhagen Prospective Personalized Oncology (CoPPO) project [26]. The aim was to study the dynamics in circulating BRAF DNA in response to BRAFi combination therapies (MEKi and EGFRi) and correlate these information's to tumor characteristics and clinical outcomes. Furthermore, we performed exome sequencing of cfDNA collected at disease progression across cancer types to identify possible resistance mutations.

\section{RESULTS}

\section{Patient characteristics}

Mutant BRAF V600E was identified in tumor tissue (fresh biopsy $n=20$, FFPE $n=3$ ) in $5 \%$ of nonmelanoma CoPPO patients (23/455 patients) including colorectal $(n=16)$, bile duct $(n=4)$, lung (Non-Small Cell
Lung Cancer, NSCLC) $(n=2)$, and pancreatic cancers $(n=1)$ (Figure 1). Seventeen patients started combination treatment with BRAFi/MEKi (lung and bile duct cancers) or BRAFi/EGFRi with or without irinotecan (colorectal cancer) (Figure 1 and Supplementary Table 2). Six patients never started treatment due to poor performance status and one patient requested early termination of treatment due to toxicity, and was therefore non-evaluable (NE). Sixteen patients were evaluable according to RECIST1.1 and cfDNA was collected at baseline and at least at 4 weeks after starting therapy from 12 of these patients. Four patients had either non-detectable cfBRAFV600E levels using ddPCR (allele fraction, AF $<0.001$ ) or no plasma sample collected at baseline (Figure 1). The latter sample was excluded from the statistical analyses on baseline cfBRAFV600E levels (Figure 3). A total of 124 plasma samples were collected from the 16 evaluable patients with a mean number of 7.75 samples per patient.

\section{Tumor response and progression}

Thirteen of the 16 evaluable patients (81\%) had reductions in their target tumor lesions on CT scans as shown by the waterfall plot (Figure 2A). Eight patients achieved an objective tumor response ( $C R \mathrm{n}=1, \mathrm{PR}$ $\mathrm{n}=7$ ), seven had SD, and one had PD as best response according to RECIST 1.1. The median PFS and OS were 4.8 and 15 months, respectively (Supplementary Figure 1). Additionally, the PFS ratio was $>1.3$ in nine patients which means that more than half of the patients experienced $>30 \%$ longer PFS on BRAFi combinations compared to the most recent treatment (Supplementary Table 2). We also assessed the treatment response in plasma cfDNA and interestingly, changes in BRAF V600E AF compared to baseline, indicated tumor response (decrease in AF) and progression (increase in AF) at 4 and 12 weeks after commencing therapy, respectively (Supplementary Figure 2). In concordance with this observation, patients with a reduction in cfBRAFV600E AF less than 50\% after 4 and 12 weeks had a significantly shorter OS and PFS compared to patients with a larger reduction $(>50 \%)$ from baseline levels (Supplementary Figure 3). Although these analyses were statistically significant, the observations should be assessed in a larger cohort, to confirm the correlations between cfBRAFV600E reduction and survival.

To investigate whether cfDNA was an early marker of tumor progression, we defined progression as the time point when an increase in cfDNA was observed and compared to the date when progression was recorded according to RECIST 1.1. Fourteen patients had tumor progression according to RECIST1.1 within the study period. Increases in cfBRAFV600E AF preceded radiological evidence of progression in 11 out of 16 cases (median 5 weeks, range 2-17 weeks, Figure 2B). The remaining patients were either progression-free at data cut-off (Pt2 and Pt12), had ND levels of cfBRAFV600E at RECIST-defined progression 
(Pt3 and Pt10) or had unchanged BRAF AF at progression compared to baseline (Pt14). Longitudinal measurements of the mutant AF's are presented for each of the 16 evaluable patients in Supplementary Figure 4.

\section{Baseline plasma DNA}

Baseline plasma samples were available from all BRAF-mutated patients except patient number 1 (Pt1) where no plasma was available before treatment start (Figure 1 and Supplementary Table 2). Plasma cfBRAFV600E was detectable using ddPCR at baseline in $81 \%(13 / 16)$ of the patients. The remaining three patients had undetectable levels of mutant BRAF (AF $<0.001)$ at baseline and at all measured time points during therapy (Pt 2, 3, 12, Supplementary Figure 4). The total cfDNA level per milliliter plasma (median $14.7 \mathrm{ng} / \mathrm{ml}$, range 3.2 - 175.5, $\mathrm{n}=16$ ) and cfBRAFV600E AF (median AF 0.079, range $\mathrm{ND}-0.39, \mathrm{n}=16$ ) indicated a correlation with tumor burden - defined as the sum of tumor measurements

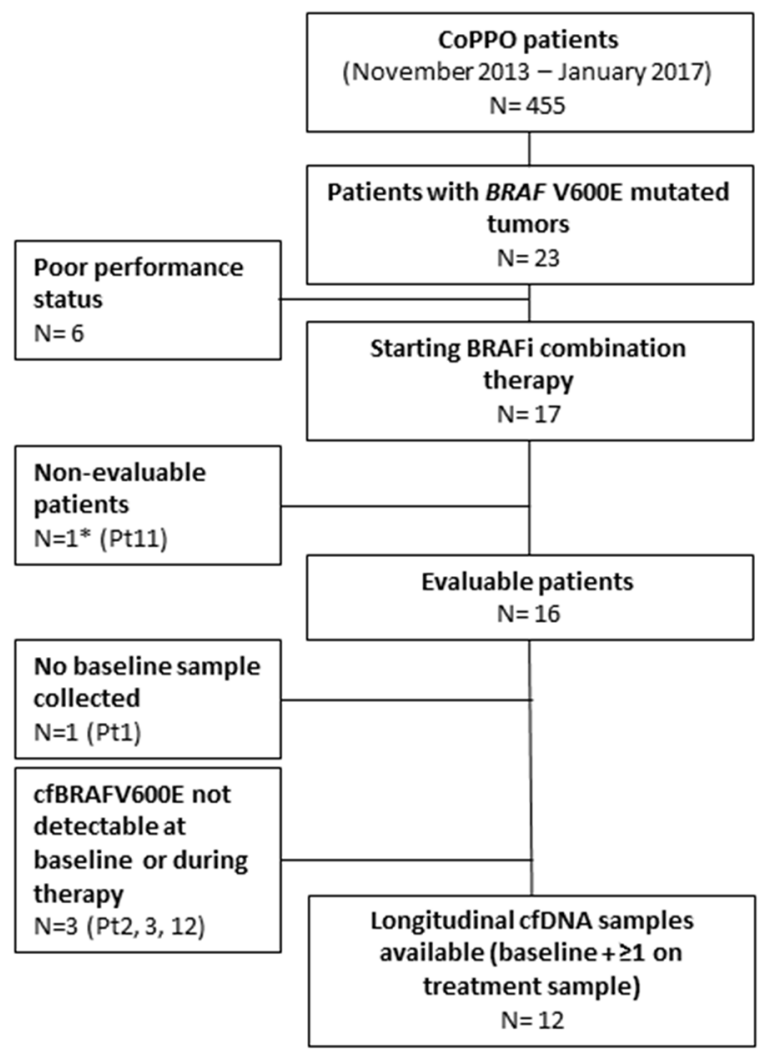

according to RECIST 1.1 (Spearman's correlation coefficient, 0.55 and $0.56 ; P<0.05$, Figure $3 \mathrm{~A}$ and $3 \mathrm{~B}$ ). We further tested whether baseline cfBRAFV600E AF $\geq 0.05$ could predict OS and PFS as shown in a similar cohort [27]. Patients with a baseline mutant fraction $\geq 0.05$ showed a non-significant trend towards shorter OS and PFS ( $p=0.098$ and $p=0.065)$ compared to those with lower levels (Figure 3C and 3D). Similar results were observed when using the median cfBRAFV600E AF (0.08) as cutpoint (data not shown).

\section{Resistance mechanisms}

Characterizing tumor evolution in response to targeted therapy is essential for understanding development of resistance. This study included a bile duct cancer patient (Pt5) who achieved a prolonged response, and exome sequencing was performed on three tissue and two plasma samples (Figure 4A-4B). The targeted BRAF mutation and a splice-site-disrupting TP53 mutation were present

\begin{tabular}{|c|c|}
\hline Characteristic & $\begin{array}{l}\text { No. of patients } \\
(n=23)\end{array}$ \\
\hline \multicolumn{2}{|l|}{ Cancer type } \\
\hline Colorectal cancer & 16 \\
\hline Bile duct cancer & 4 \\
\hline Lung cancer & 2 \\
\hline Pancreatic cancer & 1 \\
\hline \multicolumn{2}{|l|}{ Sex } \\
\hline Male & 14 \\
\hline Female & 9 \\
\hline Age-median (range) & $61(33-73)$ \\
\hline \multicolumn{2}{|l|}{ ECOG performance status } \\
\hline 0 & 10 \\
\hline 1 & 13 \\
\hline \multicolumn{2}{|l|}{ Prior lines of therapy } \\
\hline$<3$ & 11 \\
\hline$\geq 3$ & 12 \\
\hline \multicolumn{2}{|l|}{ No. of metastatic sites } \\
\hline$\leq 2$ & 4 \\
\hline$>2$ & 19 \\
\hline \multicolumn{2}{|c|}{ BRAF inhibitor combination therapy } \\
\hline Dabrafenib 1 +trametinib ${ }^{2}$ & 5 \\
\hline Vemurafenib ${ }^{1}+$ panitumumab $^{3}$ & 8 \\
\hline \multicolumn{2}{|c|}{ Vemurafenib ${ }^{1}+$ panitumumab/cetuximab ${ }^{3}+$} \\
\hline Notherapy & 6 \\
\hline
\end{tabular}

Figure 1: Study outline and baseline characteristics of patients with BRAF V600E mutated non-malignant melanoma tumors. Flow diagram (left) showing the number of patients with BRAF V600E- mutated tumors $(\mathrm{n}=23)$ and the number of patients with plasma samples collected for cfDNA analysis including baseline analyses $(n=16)$ and longitudinal evaluation $(n=12$, requiring a baseline sample and at least one plasma sample after therapy start). One patient was non-evaluable due to premature termination of therapy but the baseline plasma sample from this patient $\left(^{*}\right)$ was used for statistical analysis on baseline cfDNA levels. Characteristics of the patients with BRAF V600E-mutated tumors are shown in the table (right) including assigned therapy regimens. ${ }^{1}$ BRAF inhibitors administrated were vemurafenib $960 \mathrm{mg}$ twice a day (BID) or dabrafenib $150 \mathrm{BID} ;{ }^{2} \mathrm{MEK}$ inhibitor was trametinib $2 \mathrm{mg}$ daily (QD); ${ }^{3} \mathrm{EGRF}$ inhibitors administrated were cetuximab $500 \mathrm{mg} / \mathrm{m}^{2}$ every 14 days or panitumumab $6 \mathrm{mg} / \mathrm{kg}$ intravenously (IV) every 14 days; ${ }^{4}$ Irinotecan was administrated at a dose of $180 \mathrm{mg} / \mathrm{m}^{2}$ every 14 days. 
in all samples analyzed indicating a dominant tumor clone characterized by these two pathogenic mutations. Baseline mutant fractions were higher in the solid and liquid baseline samples compared to the samples collected at progression, likely reflecting the reduced tumor size (Figure 4A). We observed tissue- and plasma-only variants (Figure 4B and Supplementary Table 3) likely illustrating low levels of cfDNA released from a specific tumor clone and the fact that tissue biopsies only represents a fraction of the heterogeneous tumor.

Longitudinal cfBRAFV600E monitoring was compared to images of the liver metastasis, the sum of measurable lesions and cancer antigen 19-9 (CA199), and it was evident that, increases in the fraction of cfBRAFV600E indicated tumor growth $>100$ days (17 weeks) prior to other measures (Figure 4A). Furthermore, whole exome sequencing (WES) and subsequent variant analysis was performedon three cfDNA samples collected at progression from patients with metastatic colorectal cancer (Pt7, 9, and 17). We identified three variants involved in the MAPK pathway (Figure 4C and Supplementary Table 3), which were present at the time of disease progression but not detectable in baseline tumor tissue samples.

\section{DISCUSSION}

Here we show that, the fraction of BRAF V600E in cfDNA can be used to monitor the response to BRAFi combination therapy in non-melanoma cancers and importantly, indicate disease progression median five weeks before radiological evidence. Longitudinal BRAF monitoring showed that, a reduction in AF at 4 and 12 weeks after commencing treatment was significantly associated with longer PFS and OS, indicating that an early drop in cfBRAFV600E levels could be predictive of survival when monitoring advanced stage cancers using cfDNA. Of note, two patients with prolonged survival (Pt2 and Pt12) had non-detectable (ND) cfBRAFV600E levels at baseline and throughout therapy indicating, that ND levels could be a possible prognostic marker as previously suggested [18, 24]. However, these observations are promising, larger cohort studies are needed to further validate the findings. Tumor responses were also evaluated according to RECIST1.1 and a response rate of 50\% was achieved across tumor types with median PFS and OS of 4.5 and 15 months, respectively, which are comparable with previous studies [13, 28, 29]. Interestingly, we observed a partial tumor response in both patients with NSCLC and a prolonged PFS and OS compared to previously reported (median PFS 9.7 months) [30].

Despite the small cohort, baseline measurements showed a positive correlation between tumor burden and the levels of total cfDNA and cfBRAFV600E AF. Furthermore, a high baseline BRAF-fraction indicated shorter OS and PFS, an observation that was in agreement with previous reports [21, 24, 25, 27, 31-34]. In addition, we observed a high concordance (83\%) in BRAF-mutation status between tumor tissue and cfDNA possibly reflecting

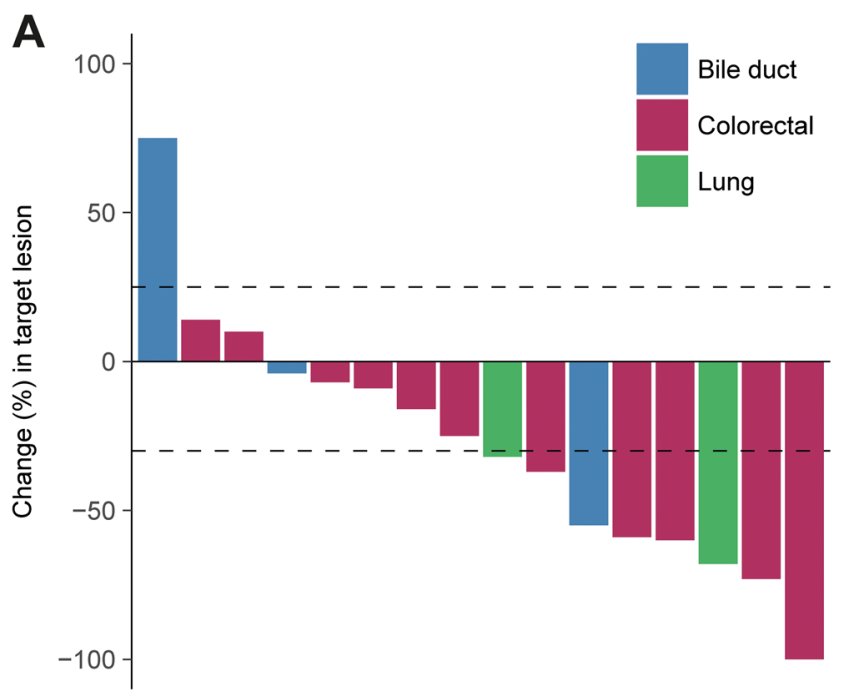

B

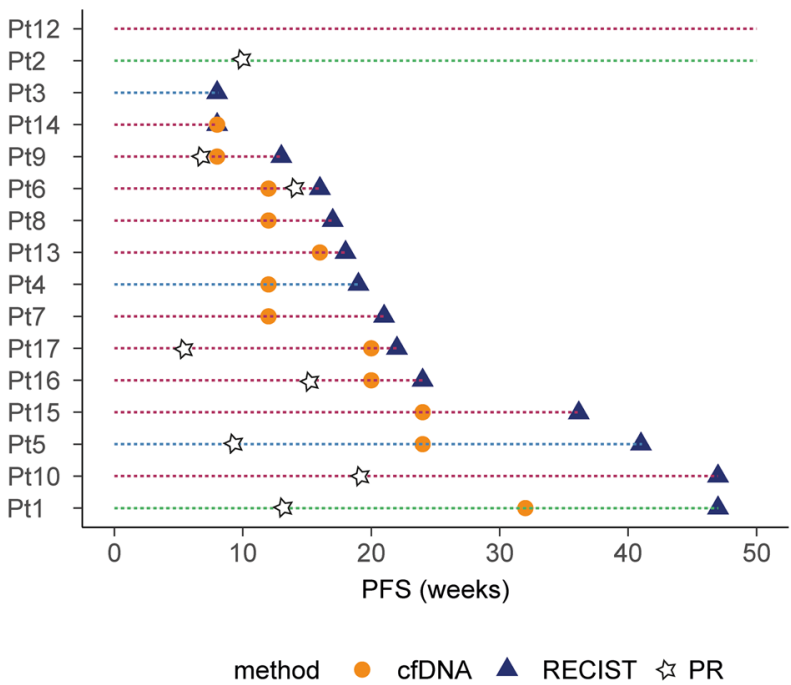

Figure 2: Tumor response and progression assessed by RECIST1.1 and cfDNA. (A) Waterfall plot showing the maximum \%-change in target tumor lesions from baseline to best response according to RECIST1.1. Changes above 20\% indicate PD, greater than $-30 \%$ (line) indicates PR, changes between $20 \%$ and $-30 \%$ indicates SD and tumor reduction of $-100 \%$ indicate CR. Each patient ( $\mathrm{n}=16$ ) is designated by a colored bar representing the cancer types as shown in the legend. (B) Disease progression assessed by cfDNA (orange circles) and RECIST1.1 (blue triangles) for each patient (indicated by a colored horizontal line). The patient ID's are indicated on the y-axis and the $\mathrm{x}$-axis show PFS in weeks. The star symbols indicate the time of best response according to RECIST1.1 for the patients with PR or CR. 
the advanced disease status and the use of fresh biopsies instead of archival tumor material [35, 36]. Previous reports have shown a concordance ranging from $57-84 \%$ primarily from melanoma studies $[12,18,20,21,24,32$, 37, 38].

This cohort was ideal for evaluating cfBRAFV600E as a marker of therapy response and resistance in non-melanoma cancers, as the cohort included extensive plasma samples and most patients developed disease recurrence within a short timescale. However, our study had some limitations including a small cohort size, heterogeneity of cancer types and treatments. Furthermore, the non-invasive follow-up strategy included only the BRAF V600E mutation which is clinically relevant to only a subset of patients and complementary NGS analysis covering other cancer hotspot genes should be considered for early discovery of driver and resistance alterations.

Resistance to BRAFi therapy constitutes a major challenge and mutations leading to re-activation of the MAPK-pathway have been described in both malignant melanoma and colorectal cancers. Investigating a broad list of 278 MAPK-related genes in cfDNA collected at progression, identified 11 variants (including BRAF V600E), of which six where novel variants and five were previously described in e.g. the COSMIC database. Functional studies are needed to clarify the molecular effect of these variants. Patient Pt9 had a high frequency MEK1 variant (c.173A>C, p.Q58P, Table S3) located in a conserved region where several alterations have been reported in BRAFi-resistant melanoma samples [39] indicating that, this region might be involved in resistance
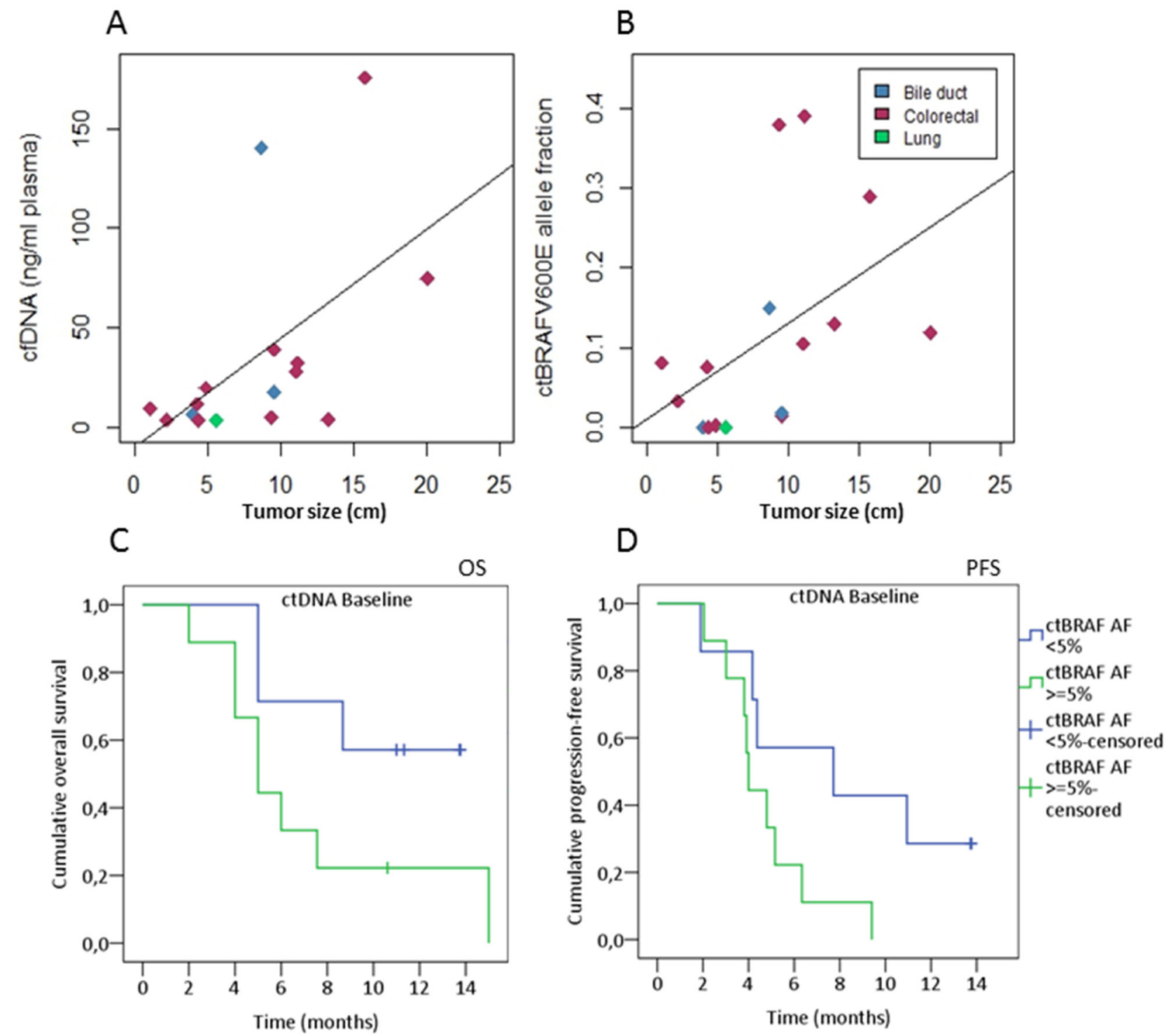

Figure 3: Baseline cfDNA levels correlate with tumor size and survival. (A) A positive correlation was observed between the sum of target tumor measures according to RECIST 1.1 and total cfDNA levels and cfBRAFV600E allele fraction (B). The Spearman's rank correlation coefficient was $0.55(\mathrm{p}=0.026)$ and $0.56(\mathrm{p}=0.024)$, respectively. Patients having both a CT scan and a baseline cfDNA sample were included $(\mathrm{n}=16)$. Cancer types are indicated by color as shown in the legend. High baseline ctBRAFV600E AF was correlated to shorter OS (C) ( $\mathrm{n}=16, \mathrm{p}=0.098$, log-rank test) and PFS (D) $(\mathrm{n}=16, P=0.065$, log-rank test) in patients with BRAF V600E-mutated cancers stratified on the level of baseline ctBRAFV600E AF threshold of 0.05 ( $5 \%$ allele frequency). 
but functional tests are required. Additionally, WES of multiple tissue and plasma biopsies from a bile duct cancer patient (Pt5) illustrated a dominant tumor clone characterized by a pathogenic TP53 mutation and the treatment target BRAF V600E detected in all solid and liquid biopsies, possibly explaining the good response observed for this patient. Interestingly, progression-only variants were identified in both tissue and plasma DNA but none of the variants were overlapping, possibly illustrating the challenges associated with tumor clonality or differences in sequencing depth and thus allele frequency.

In conclusion, our study indicates that BRAFi combination therapy is effective in advanced, solid non-melanoma BRAFV600E-mutated cancers and cfBRAFV600E may be used as an early indicator of response and progression.
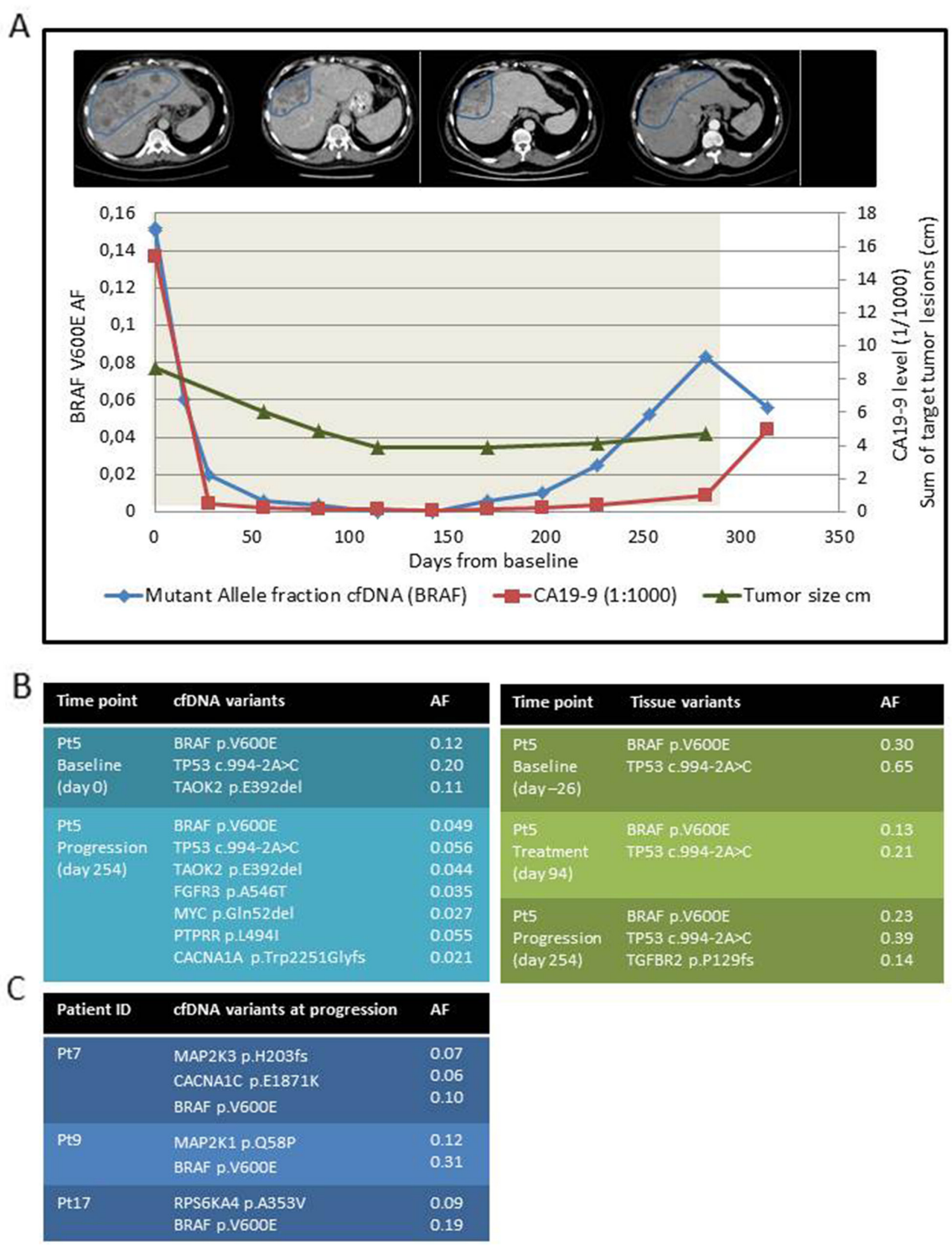

Figure 4: Monitoring tumor response and MAPK-related mutations in response to BRAFi combination therapy. Panel (A) illustrates the tumor response in a bile duct cancer patient (Pt5). At the top, the tumor response assessed by CT scans marking the area with liver metastasis (blue marking). Below, the changes in the sum of target tumor lesions according to RECIST 1.1(green line), CA19-9 levels (red line) and cfBRAFV600E AF (blue line) are shown. The x-axis indicates the time from therapy start (day 0) until collection of the last plasma sample (day 321) including the day of therapy termination due to progression (day 286, end of grey shading). The primary $\mathrm{y}$-axis indicates the BRAF V600E AF and the secondary y-axis shows both the CA19-9 level (presented as divided by 1000 due to an extreme baseline value of $>16000$ ) and the sum of measurable tumor lesions according to RECIST 1.1. Panel (B) shows the MAPKassociated variants identified in the three tissue biopsies (green table) and the two plasma samples (blue table). Due to the difference in sequencing depth and therefore mutation fraction cut-offs (tissue $\mathrm{AF} \geq 0.10$, plasma $\mathrm{AF} \geq 0.02$ ) we were not able to directly compare tissue with plasma mutations. Table (C) summarizes the progression-only MAPK-related variants together with the BRAF V600E mutation identified in cfDNA from three colorectal cancer patients with early progression and a high baseline cfBRAFV600E AF. 


\section{MATERIALS AND METHODS}

\section{Patients and sample collection}

Fresh tumor biopsies were collected and molecularly characterized in the CoPPO study (NCT02290522) as previously described [26]. Formalin-fixed paraffinembedded (FFPE) archival tumor tissue or plasma cfDNA was examined in cases where tumors were non-accessible for biopsy. All patients had metastatic solid tumors and exhausted treatment options. Studies were conducted in accordance with the Declaration of Helsinki and written informed consent was obtained for all patients (Danish Ethical Committee, file number: 1300530). Patients were allocated to targeted treatment according to recommendations made by a multidisciplinary tumor board. Computed Tomography (CT) scans were performed every eight weeks and tumor responses were evaluated according to Response Evaluation Criteria In Solid Tumors (RECIST) 1.1 [40] and classified as complete response (CR), partial response (PR), stable disease (SD) or progressive disease (PD). The PFS ratio was calculated as PFS on BRAFi combination treatment divided by PFS on most recent treatment. A PFS ratio $>1.3$ was reported because an improvement in PFS $>30 \%$ has been described to be a clinical meaningful cut-off [41]. Plasma samples for cfDNA analysis were collected immediately before treatment initiation (within 24h), longitudinally every four weeks, and if possible, at progression or after treatment termination.

\section{DNA extraction and quantification}

Peripheral blood was collected in BCT tubes (Streck Laboratories, Omaha, NE, USA) as previously described [42]. Circulating DNA was extracted from 2-4 ml plasma using the QIAsymphony Circulating DNA Kit (Qiagen, Hilden, Germany) according to the manufacturer's instructions using an elution volume of $60 \mu \mathrm{l}$. Extracted cfDNA was stored at $-20^{\circ} \mathrm{C}$ until further use. DNA quantification was performed using a dsDNA HS Assay Kit (Thermo Fisher Scientific, Waltham, MA) on a Qubit Fluorometer (Thermo Fisher Scientific, Waltham, MA) and the concentration of cfDNA per milliliter of plasma was calculated for each time point.

\section{Droplet digital PCR}

Mutant BRAF V600E was quantified using the QX200 droplet digital PCR (ddPCR) system from Bio-Rad (Bio-Rad/Molecular MD, California, USA). Dual labeled (FAM or HEX) fluorescent probes for BRAF V600E and the wild type loci were used (BioRad, V600E; cat no. 10031246 dHsaCP2000027, WT for V600E; cat no. 10031249 dHsaCP2000028). PCR reaction mixtures were run and subsequently analyzed using the QX200 reader, according to manufactures instructions. The mutant allele fraction (AF, mutant counts/total DNA counts (mutant + wild type)) was estimated using QuantaSoft v.1.7.4 software from Bio-Rad. Mutant AFs $\geq 0.001$ (0.1\%) were detected requiring three or more mutation-positive droplets per well as recommended by the company. An increase in cfBRAFV600E AF was recorded if the AF increased from non-detectable to detectable levels ( $\mathrm{AF} \geq 0.001$ ) or increased in two consecutive samples unless it was the last plasma sample collected prior to termination of treatment due to clinical progression.

\section{Exome sequencing}

Whole exome sequencing was performed on plasma cfDNA samples (Pt5, 7, 9 and 17) obtained at disease progression from patients with a high $\mathrm{AF}$ of cfBRAFV600E. Patient tumor samples were also analyzed by WES as part of the CoPPO study [26]. DNA libraries were constructed from 10 ng cfDNA using NEBNext Ultra II DNA Library Prep kit for Illumina protocols (version July 2016, New England Biolabs). Hybridization-based exome capture was performed according to the manufacturer's instructions using the SeqCap EZ MEDExome (Roche NimbleGen Inc.). The samples were pooled and sequenced on the Illumina NextSeq platform to a minimum average coverage $>50 \mathrm{x}$. Analysis of WES data from both tumor tissue DNA and cfDNA was performed using the same pipeline including the Qiagen software Biomedical Genomics Workbench version 3.0 and Ingenuity Variant Analysis version 5.1. Briefly, confident calls originating from bidirectional sequence reads were filtered by a phred quality score $\geq 30$, read depth of $\geq 20$, and allele frequency $\geq 5 \%$. Tumor-specific variants were selected by subtracting the germline variants which were available through the CoPPO project [26]. Ingenuity Variant Analysis was used to identify coding or splice site disrupting variants $(+/-2$ base pairs from exonintron boundaries). Common variants reported with an allele frequency $>1 \%$ in the 1000 genomes project, the NHLBI ESP exomes, or the ExAC frequency were removed, unless the variant was established as a common pathogenic variant according to the Ingenuity Knowledge database. Variant analysis was broadly focused on the MAPK pathways using a comprehensive gene list from Gene Set Enrichment Analysis (GESA) including genes from the classical MAP kinase pathway as well as the JNK/p38 and ERK5 pathways (M10792; KEGG_MAPK_SIGNALING_PATHWAY) [43, 44] (Supplementary Table 1). In addition, an extended list of cancer-associated variants identified by exome sequencing is included in (Supplementary Table 4). The variant lists were generated using the Ingenuity Variant Analysis software. 


\section{Statistical analyses}

Overall survival (OS) and PFS was estimated using Kaplan-Meier statistics [45] and a log-rank test was used to compare OS and PFS among patient groups. PFS was defined, as the time from treatment start to disease progression (according to RECIST 1.1 or clinical evaluation) or death. Overall survival was defined as the time from treatment start to date of death. Patients that were progression-free or still alive at the day of data cut-off (24 October 2017) were censored in the survival analyses. The Spearman rank correlation analysis was used to compare tumor and plasma measures. All analyses were two-sided, and $P$-values $<0.05$ were considered statistically significant. Statistical analyses were performed using IBM Statistics SPSS (version 22) and R (version 0.99.903).

\section{Abbreviations}

cfDNA: Cell-free DNA; ddPCR: Droplet digital PCR; NGS: Next generation sequencing; BRAFi: BRAF inhibitor; MEKi: MEK inhibitor; EGFRi: EGFR inhibitor; RECIST: Response evaluation criteria in solid tumors; CR: Complete response; PR: Partial response; SD: Stable disease; PD: Progressive disease; BID: twice a day; QD: Daily; AF: Allele fraction.

\section{Author contributions}

The study was designed and supervised by MMS, $\mathrm{OO}$ and CWY. Analysis and interpretation of data was performed by LBA who also wrote the manuscript. IVT provided clinical data on the patients. KSR and MMS enrolled patients in the CoPPO study headed by FCN, ESR and UL. FM, AYS and SK provided technical support related to the exome sequencing.

\section{ACKNOWLEDGMENTS}

We sincerely thank patients and staff at the Phase I unit and Centre for Genomic Medicine (Rigshospitalet). A special thanks to Mette Madsen, Cecilia Brunhoff Haakansson, Katarina Larsen, Miriam Yan Juk Guo, Aseeba Ayub, Line Offenbach Jacobsen, and Bettina Marsot for technical assistance and to Nitzan Rosenfeld and James Morris for collaboration on the WES data.

\section{CONFLICTS OF INTEREST}

Eric Santoni-Rugiu has received lecture honoraria from Roche, Pfizer, Novartis, and Boehringer Ingelheim. Morten Mau-Sørensen has received lecture honoraria and support to participate in scientific conference from Roche. All other authors declare no potential conflicts of interest.

\section{FUNDING}

This study was supported by the Danish Cancer Society, The Harboe Foundation, and the Oncological Research Fund, Department of Oncology, Copenhagen University Hospital, Denmark. The sponsors had no role in study design, data analysis, or writing of the article.

\section{REFERENCES}

1. Davies H, Bignell GR, Cox C, Stephens P, Edkins S, Clegg S, Teague J, Woffendin H, Garnett MJ, Bottomley W, Davis N, Dicks E, Ewing R, et al. Mutations of the BRAF gene in human cancer. Nature. 2002; 417: 949-54. https://doi.org/10.1038/nature00766.

2. Curtin JA, Fridlyand J, Kageshita T, Patel HN, Busam KJ, Kutzner H, Cho KH, Aiba S, Brocker EB, LeBoit PE, Pinkel D, Bastian BC. Distinct sets of genetic alterations in melanoma. $\mathrm{N}$ Engl J Med. 2005; 353: 2135-47. https://doi.org/10.1056/NEJMoa050092.

3. De Roock W, Claes B, Bernasconi D, De Schutter J, Biesmans B, Fountzilas G, Kalogeras KT, Kotoula V, Papamichael D, Laurent-Puig P, Penault-Llorca F, Rougier P, Vincenzi B, et al. Effects of KRAS, BRAF, NRAS, and PIK3CA mutations on the efficacy of cetuximab plus chemotherapy in chemotherapy-refractory metastatic colorectal cancer: a retrospective consortium analysis. Lancet Oncol. 2010; 11: 753-62. https://doi.org/10.1016/S1470-2045(10)70130-3.

4. Van Cutsem E, Kohne CH, Lang I, Folprecht G, Nowacki MP, Cascinu S, Shchepotin I, Maurel J, Cunningham D, Tejpar S, Schlichting M, Zubel A, Celik I, et al. Cetuximab plus irinotecan, fluorouracil, and leucovorin as firstline treatment for metastatic colorectal cancer: updated analysis of overall survival according to tumor KRAS and BRAF mutation status. J Clin Oncol. 2011; 29: 2011-9. https://doi.org/10.1200/JCO.2010.33.5091.

5. Kimura ET, Nikiforova MN, Zhu Z, Knauf JA, Nikiforov YE, Fagin JA. High prevalence of BRAF mutations in thyroid cancer: genetic evidence for constitutive activation of the RET/PTC-RAS-BRAF signaling pathway in papillary thyroid carcinoma. Cancer Res. 2003; 63: 1454-7.

6. Kris MG, Johnson BE, Berry LD, Kwiatkowski DJ, Iafrate AJ, Wistuba II, Varella-Garcia M, Franklin WA, Aronson SL, Su PF, Shyr Y, Camidge DR, Sequist LV, et al. Using multiplexed assays of oncogenic drivers in lung cancers to select targeted drugs. JAMA. 2014; 311: 1998-2006. https://doi.org/10.1001/jama.2014.3741.

7. Sanchez-Torres JM, Viteri S, Molina MA, Rosell R. BRAF mutant non-small cell lung cancer and treatment with BRAF inhibitors. Transl Lung Cancer Res. 2013; 2: 244 50. https://doi.org/10.3978/j.issn.2218-6751.2013.04.01.

8. Tiacci E, Trifonov V, Schiavoni G, Holmes A, Kern W, Martelli MP, Pucciarini A, Bigerna B, Pacini R, Wells VA, Sportoletti P, Pettirossi V, Mannucci R, et al. BRAF 
mutations in hairy-cell leukemia. N Engl J Med. 2011; 364: 2305-15. https://doi.org/10.1056/NEJMoa1014209.

9. Goeppert B, Frauenschuh L, Renner M, Roessler S, Stenzinger A, Klauschen F, Warth A, Vogel MN, Mehrabi A, Hafezi M, Boehmer K, von Deimling A, Schirmacher P, et al. BRAF V600E-specific immunohistochemistry reveals low mutation rates in biliary tract cancer and restriction to intrahepatic cholangiocarcinoma. Mod Pathol. 2014; 27: 1028-34. https://doi.org/10.1038/modpathol.2013.206.

10. Chapman PB, Hauschild A, Robert C, Haanen JB, Ascierto P, Larkin J, Dummer R, Garbe C, Testori A, Maio M, Hogg D, Lorigan P, Lebbe C, et al, and BRIM-3 Study Group. Improved survival with vemurafenib in melanoma with BRAF V600E mutation. N Engl J Med. 2011; 364: 2507-16. https://doi.org/10.1056/NEJMoa1103782.

11. Flaherty KT, Robert C, Hersey P, Nathan P, Garbe C, Milhem M, Demidov LV, Hassel JC, Rutkowski P, Mohr P, Dummer R, Trefzer U, Larkin JM, et al, and METRIC Study Group. Improved survival with MEK inhibition in BRAFmutated melanoma. N Engl J Med. 2012; 367: 107-14. https://doi.org/10.1056/NEJMoa1203421.

12. Hyman DM, Puzanov I, Subbiah V, Faris JE, Chau I, Blay JY, Wolf J, Raje NS, Diamond EL, Hollebecque A, Gervais R, Elez-Fernandez ME, Italiano A, et al. Vemurafenib in Multiple Nonmelanoma Cancers with BRAF V600 Mutations. N Engl J Med. 2015; 373: 72636. https://doi.org/10.1056/NEJMoa1502309.

13. Yaeger R, Cercek A, O'Reilly EM, Reidy DL, Kemeny N, Wolinsky T, Capanu M, Gollub MJ, Rosen N, Berger MF, Lacouture ME, Vakiani E, Saltz LB. Pilot trial of combined BRAF and EGFR inhibition in BRAF-mutant metastatic colorectal cancer patients. Clin Cancer Res. 2015; 21: 1313-20. https://doi.org/10.1158/1078-0432.CCR-14-2779.

14. Johannessen CM, Boehm JS, Kim SY, Thomas SR, Wardwell L, Johnson LA, Emery CM, Stransky N, Cogdill AP, Barretina J, Caponigro G, Hieronymus H, Murray RR, et al. COT drives resistance to RAF inhibition through MAP kinase pathway reactivation. Nature. 2010; 468: 968-72. https://doi.org/10.1038/nature09627.

15. Shi H, Hugo W, Kong X, Hong A, Koya RC, Moriceau G, Chodon T, Guo R, Johnson DB, Dahlman KB, Kelley MC, Kefford RF, Chmielowski B, et al. Acquired resistance and clonal evolution in melanoma during BRAF inhibitor therapy. Cancer Discov. 2014; 4: 80-93. https://doi.org/10.1158/2159-8290.CD-13-0642.

16. Wan JCM, Massie C, Garcia-Corbacho J, Mouliere F, Brenton JD, Caldas C, Pacey S, Baird R, Rosenfeld N. Liquid biopsies come of age: towards implementation of circulating tumour DNA. Nat Rev Cancer. 2017; 17: 223 38. https://doi.org/10.1038/nrc.2017.7.

17. Cohen JD, Li L, Wang Y, Thoburn C, Afsari B, Danilova L, Douville C, Javed AA, Wong F, Mattox A, Hruban RH, Wolfgang CL, Goggins MG, et al. Detection and localization of surgically resectable cancers with a multi-analyte blood test. Science. 2018; 359: 926-30. https://doi.org/10.1126/science.aar3247.

18. Santiago-Walker A, Gagnon R, Mazumdar J, Casey M, Long GV, Schadendorf D, Flaherty K, Kefford R, Hauschild A, Hwu P, Haney P, O'Hagan A, Carver J, et al. Correlation of BRAF Mutation Status in Circulating-Free DNA and Tumor and Association with Clinical Outcome across Four BRAFi and MEKi Clinical Trials. Clin Cancer Res. 2016; 22: 56774. https://doi.org/10.1158/1078-0432.CCR-15-0321.

19. Diehl F, Schmidt K, Choti MA, Romans K, Goodman S, Li M, Thornton K, Agrawal N, Sokoll L, Szabo SA, Kinzler KW, Vogelstein B, Diaz LA Jr. Circulating mutant DNA to assess tumor dynamics. Nat Med. 2008; 14: 985-90. https://doi.org/10.1038/nm.1789.

20. Sanmamed MF, Fernandez-Landazuri S, Rodriguez C, Zarate R, Lozano MD, Zubiri L, Perez-Gracia JL, MartinAlgarra S, Gonzalez A. Quantitative cell-free circulating BRAFV600E mutation analysis by use of droplet digital PCR in the follow-up of patients with melanoma being treated with BRAF inhibitors. Clin Chem. 2015; 61: 297304. https://doi.org/10.1373/clinchem.2014.230235.

21. Gray ES, Rizos H, Reid AL, Boyd SC, Pereira MR, Lo J, Tembe V, Freeman J, Lee JH, Scolyer RA, Siew K, Lomma C, Cooper A, et al. Circulating tumor DNA to monitor treatment response and detect acquired resistance in patients with metastatic melanoma. Oncotarget. 2015; 6: 42008-18. https://doi.org/10.18632/oncotarget.5788.

22. Tsao SC, Weiss J, Hudson C, Christophi C, Cebon J, Behren A, Dobrovic A. Monitoring response to therapy in melanoma by quantifying circulating tumour DNA with droplet digital PCR for BRAF and NRAS mutations. Sci Rep. 2015; 5: 11198. https://doi.org/10.1038/srep11198.

23. Xi L, Pham TH, Payabyab EC, Sherry RM, Rosenberg SA, Raffeld M. Circulating Tumor DNA as an Early Indicator of Response to T-cell Transfer Immunotherapy in Metastatic Melanoma. Clin Cancer Res. 2016; 22: 5480-6. https://doi.org/10.1158/1078-0432.CCR-16-0613.

24. Janku F, Huang HJ, Claes B, Falchook GS, Fu S, Hong D, Ramzanali NM, Nitti G, Cabrilo G, Tsimberidou AM, Naing A, Piha-Paul SA, Wheler JJ, et al. BRAF Mutation Testing in Cell-Free DNA from the Plasma of Patients with Advanced Cancers Using a Rapid, Automated Molecular Diagnostics System. Mol Cancer Ther. 2016; 15: 1397-404. https://doi.org/10.1158/1535-7163.MCT-15-0712.

25. El Messaoudi S, Mouliere F, Du Manoir S, BascoulMollevi C, Gillet B, Nouaille M, Fiess C, Crapez E, Bibeau F, Theillet C, Mazard T, Pezet D, Mathonnet $\mathrm{M}$, et al. Circulating DNA as a Strong Multimarker Prognostic Tool for Metastatic Colorectal Cancer Patient Management Care. Clin Cancer Res. 2016; 22: 3067-77. https://doi.org/10.1158/1078-0432.CCR-15-0297.

26. Tuxen IV, Jonson L, Santoni-Rugiu E, Hasselby JP, Nielsen FC, Lassen U. Personalized oncology: genomic 
screening in phase 1. APMIS. 2014; 122: 723-33. https://doi.org/10.1111/apm.12293.

27. Schwaederle M, Husain H, Fanta PT, Piccioni DE, Kesari S, Schwab RB, Patel SP, Harismendy O, Ikeda M, Parker BA, Kurzrock R. Use of Liquid Biopsies in Clinical Oncology: Pilot Experience in 168 Patients. Clin Cancer Res. 2016; 22: 5497-505. https://doi.org/10.1158/1078-0432.CCR-16-0318.

28. Lavingia V, Fakih M. Impressive response to dual BRAF and MEK inhibition in patients with BRAF mutant intrahepatic cholangiocarcinoma-2 case reports and a brief review. J Gastrointest Oncol. 2016; 7: E98-E102. https://doi.org/10.21037/jgo.2016.09.13.

29. Loaiza-Bonilla A, Clayton E, Furth E, O'Hara M, Morrissette J. Dramatic response to dabrafenib and trametinib combination in a BRAF V600E-mutated cholangiocarcinoma: implementation of a molecular tumour board and next-generation sequencing for personalized medicine. Ecancermedicalscience. 2014; 8: 479. https://doi.org/10.3332/ecancer.2014.479.

30. Planchard D, Besse B, Groen HJM, Souquet PJ, Quoix E, Baik CS, Barlesi F, Kim TM, Mazieres J, Novello S, Rigas JR, Upalawanna A, D'Amelio AM Jr, et al. Dabrafenib plus trametinib in patients with previously treated BRAF(V600E)mutant metastatic non-small cell lung cancer: an open-label, multicentre phase 2 trial. Lancet Oncol. 2016; 17: 984-93. https://doi.org/10.1016/S1470-2045(16)30146-2.

31. Phallen J, Sausen M, Adleff V, Leal A, Hruban C, White J, Anagnostou V, Fiksel J, Cristiano S, Papp E, Speir S, Reinert T, Orntoft MW, et al. Direct detection of earlystage cancers using circulating tumor DNA. Sci Transl Med. 2017; 9. https://doi.org/10.1126/scitranslmed.aan2415.

32. Ascierto PA, Minor D, Ribas A, Lebbe C, O'Hagan A, Arya N, Guckert M, Schadendorf D, Kefford RF, Grob JJ, Hamid O, Amaravadi R, Simeone E, et al. Phase II trial (BREAK2) of the BRAF inhibitor dabrafenib (GSK2118436) in patients with metastatic melanoma. J Clin Oncol. 2013; 31: 3205-11. https://doi.org/10.1200/JCO.2013.49.8691.

33. Kim K, Shin DG, Park MK, Baik SH, Kim TH, Kim S, Lee S. Circulating cell-free DNA as a promising biomarker in patients with gastric cancer: diagnostic validity and significant reduction of cfDNA after surgical resection. Ann Surg Treat Res. 2014; 86: 136-42. https://doi.org/10.4174/astr.2014.86.3.136.

34. Sozzi G, Conte D, Leon M, Ciricione R, Roz L, Ratcliffe C, Roz E, Cirenei N, Bellomi M, Pelosi G, Pierotti MA, Pastorino U. Quantification of free circulating DNA as a diagnostic marker in lung cancer. J Clin Oncol. 2003; 21: 3902-8. https://doi.org/10.1200/JCO.2003.02.006.

35. Siravegna G, Marsoni S, Siena S, Bardelli A. Integrating liquid biopsies into the management of cancer. Nat Rev Clin Oncol. 2017; 14: 531-48. https://doi.org/10.1038/nrclinonc.2017.14.

36. Siravegna G, Mussolin B, Buscarino M, Corti G, Cassingena A, Crisafulli G, Ponzetti A, Cremolini C,
Amatu A, Lauricella C, Lamba S, Hobor S, Avallone A, et al. Clonal evolution and resistance to EGFR blockade in the blood of colorectal cancer patients. Nat Med. 2015; 21: 795-801. https://doi.org/10.1038/nm.3870.

37. Bettegowda C, Sausen M, Leary RJ, Kinde I, Wang Y, Agrawal N, Bartlett BR, Wang H, Luber B, Alani RM, Antonarakis ES, Azad NS, Bardelli A, et al. Detection of circulating tumor DNA in early- and late-stage human malignancies. Sci Transl Med. 2014; 6: 224 ra24. https://doi.org/10.1126/scitranslmed.3007094.

38. Gonzalez-Cao M, Mayo-de-Las-Casas C, Molina-Vila MA, De Mattos-Arruda L, Munoz-Couselo E, Manzano JL, Cortes J, Berros JP, Drozdowskyj A, Sanmamed M, Gonzalez A, Alvarez C, Viteri S, et al. BRAF mutation analysis in circulating free tumor DNA of melanoma patients treated with BRAF inhibitors. Melanoma Res. 2015; 25: 486-95.

39. Van Allen EM, Wagle N, Sucker A, Treacy DJ, Johannessen CM, Goetz EM, Place CS, Taylor-Weiner A, Whittaker S, Kryukov GV, Hodis E, Rosenberg M, McKenna A, et al, and Dermatologic Cooperative Oncology Group of Germany (DeCOG). The genetic landscape of clinical resistance to RAF inhibition in metastatic melanoma. Cancer Discov. 2014; 4: 94-109. https://doi.org/10.1158/2159-8290.CD-13-0617.

40. Eisenhauer EA, Therasse P, Bogaerts J, Schwartz LH, Sargent D, Ford R, Dancey J, Arbuck S, Gwyther S, Mooney M, Rubinstein L, Shankar L, Dodd L, et al. New response evaluation criteria in solid tumours: revised RECIST guideline (version 1.1). Eur J Cancer. 2009; 45: 228-47. https://doi.org/10.1016/j.ejca.2008.10.026.

41. Mick R, Crowley JJ, Carroll RJ. Phase II clinical trial design for noncytotoxic anticancer agents for which time to disease progression is the primary endpoint. Control Clin Trials. 2000; 21: 343-59.

42. Ahlborn LB, Madsen M, Jonson L, Nielsen FC, Lassen U, Yde CW, Mau-Sorensen M. Concordance of Mutation Detection in Circulating Tumor DNA in Early Clinical Trials Using Different Blood Collection Protocols. Clin Lab. 2017; 63: 1755-9. https://doi.org/10.7754/Clin.Lab.2017.170516.

43. Liberzon A, Birger C, Thorvaldsdottir H, Ghandi M, Mesirov JP, Tamayo P. The Molecular Signatures Database (MSigDB) hallmark gene set collection. Cell Syst. 2015; 1: 417-25. https://doi.org/10.1016/j.cels.2015.12.004.

44. Subramanian A, Tamayo P, Mootha VK, Mukherjee S, Ebert BL, Gillette MA, Paulovich A, Pomeroy SL, Golub TR, Lander ES, Mesirov JP. Gene set enrichment analysis: a knowledge-based approach for interpreting genome-wide expression profiles. Proc Natl Acad Sci U S A. 2005; 102 : 15545-50. https://doi.org/10.1073/pnas.0506580102.

45. Goel MK, Khanna P, Kishore J. Understanding survival analysis: Kaplan-Meier estimate. Int J Ayurveda Res. 2010; 1: 274-8. https://doi.org/10.4103/0974-7788.76794. 